\title{
The Benefit, Risks and Cost of Primary Stroke Prevention with Antihypertensive Drugs
}

\author{
Christopher J. Bulpitt
}

\begin{abstract}
The benefits of treatment with anti-hypertensive drugs not only include a reduction in stroke incidence of $40 \%$ but also a reduction in cardiac deaths, severe congestive heart failure, severe retinal changes and almost certainly a reduction in the incidence of angina. The risks depend on the drug employed and include gout and diabetes mellitus for diuretics and symptom side effects with all drugs. Certain drugs have been proven to impair the quality of life of treated patients. The cost of an extra year of life gained is lower in men, in the elderly and at higher levels of diastolic pressure. It is desirable to adjust the years of life gained by their quality (a cost-utility analysis) but such methods await good data. (Hypertens Res 1994; 17 Suppl. I: S115-S119)
\end{abstract}

Key Words: stroke prevention, anti-hypertensive drugs, adverse drug reactions, quality of life, costeffectiveness

The best definition of hypertension is "the level of blood pressure above which treatment does more good than harm". This definition has to be qualified by stating the level of systolic or diastolic pressure, the fact that the level should be a sustained and not transient reading, and a statement made as to whether or not the definition applies to both sexes and all ages. In order to define the benefit and risks of antihypertensive treatment we have to look at the results of randomised controlled trials.

\section{Benefits}

Collins and his colleagues (1) have performed a meta-analysis of the trial data and concluded that antihypertensive treatment reduces stroke events by an average of $42 \%$ (range $33-50 \%$ for the trials). They concluded that epidemiological data would suggest a reduction of $35-40 \%$ for the equivalent reduction in blood pressure and therefore the Fraction of Benefit (FOB) obtained was $100 \%$. In the elderly a meta-analysis by Amery et al. (2) suggested that stroke events in the trials were associated with a $40 \%$ reduction, thus confirming a similar efficacy of treatment in the elderly. The consistency of the trial data has led many authorities to assume that it is the blood pressure lowering effect of antihypertensive drugs that confirms the reduction in stroke incidence and that all drugs should be equally efficacious if they reduce pressure by a given amount. Nevertheless, stroke events were not reduced by propranolol in smokers in the first Medical Research Council trial in middle aged subjects (3), whereas bendrofluazide did reduce the risk.
This interaction between smoking and treatment has not been supported for stroke incidence in the International Prospective Primary Study in Hypertension (IPPPSH) trial (4), nor in the Heart Attack Primary Prevention in Hypertension (HAPPHY) trial (5).

When considering the benefits of antihypertensive treatment for strokes it is also important to consider other benefits that may accrue. Collins et al. (1) also performed a meta-analysis on the reduction in coronary events during the trials. The reduction averaged $14 \%$, range $4-22 \%$, with a FOB of $60 \%$. Thus the gains predicted for coronary heart disease from epidemiological data are not fully realised from antihypertensive treatment. Two possible reasons have been widely discussed, that a lag time should be considered before any benefits are realised and secondly that the drugs employed may have an adverse effect on serum lipids and thus prevent the benefits being fully realised. In the MRC trial in younger subjects there was evidence for a reduction in coronary events with propranolol in men who did not smoke. This was not observed in men who smoked nor men who received bendrofluazide (3). In the elderly, the MRC trial found a reduction in cardiac events of $19 \%$ (6) and other trials reported a benefit ranging from a reduction of $13 \%$ to $27 \%(7)$. The smallest reductions were observed when a beta blocker was employed as first line treatment.

In the MRC elderly trial coronary events were reduced by $44 \%$ with a hydrochorothiazide/amiloride combination but only by $3 \%$ with atenolol (6). In other studies employing mainly a beta blocker the

From the Division of Geriatric Medicine, Department of Medicine, Royal Postgraduate Medical School, London, U.K. Address for Reprints: Division of Geriatric Medicine, Department of Medicine, Royal Postgraduate Medical School, Du Cane Road, London W 12 ONN, U.K. 
reductions in cardiac event were 15\% (the HEP trial, 8), and 13\% (the STOP trial, 9). The corresponding reductions when a diuretic was employed were $19 \%$ (Australian trial, 10), 20\%(EWPHE trial, 11) and 27\% (SHEP trial, 12). In view of these findings, Beard et al. (7) recommended diuretics rather than beta blockers for elderly patients with uncomplicated hypertension.

The other benefits of treatment are less easy to quantify. In younger subjects accelerated hypertension with retinal haemorrhages and exudate is prevented by active treatment. Similarly it is expected that renal failure, hypertensive heart failure and multi-infarct dementia may be prevented by antihypertensive treatment. These events are infrequent and therefore the benefits of treatment are difficult to establish, especially within narrow quantitative boundaries. In the EWPHE trial (11) severe congestive heart failure was reduced by $8 / 1,000$ patient years, severe retinal changes by 4/1,000 patient years (Table 1). Pulmonary embolus was not reduced by treatment and renal failure was rare, only one death occurring in the trial (this patient was taking active treatment). Anti-hypertensive treatment may also be expected to reduce angina and the need for a beta blocker was reduced by $4 / 1,000$ patient years by active treatment (11). The prescription of a beta blocker, however, may not always have been for angina. The benefits of treatment for the EWPHE trial are summarised in Table 1.

\section{Risks}

The risks of treatment depend on the exact nature of the anti-hypertensive treatment and range from potentially life threatening events, to biochemical changes not known to be associated with symptoms, and to adverse effects on quality of life. The risks in

Table 1. Differences between Active and Placebo Treatment in the EWPHE Trial (11)

\begin{tabular}{lc}
\hline Benefit & Numbers/1,000/year \\
Fatal cardiac events & $-11^{*}$ \\
Fatal cerebrovascular events & -6 \\
Non-fatal cerebrovascular events & $-11^{*}$ \\
Severe congestive cardiac failure & $-8^{*}$ \\
Severe retinal changes & -4 \\
Need for beta blocker & -4 \\
\hline Risk & \\
Gout & $+4^{*}$ \\
Mild hypokalaemia [K+<3.5 mmol/l] & $+71^{* *}$ \\
Elevated serum creatinine [ $\geq 180 \mu \mathrm{mol} / 1]$ & $+23^{* *}$ \\
Diabetes & +9 \\
Dry mouth & $+124^{* *}$ \\
Diarrhoea & $+71^{*}$ \\
Need for hypoglycaemic drugs & +7 \\
Need for peripheral vasodilators & +7 \\
\hline$* p<0.05, * * p 0.001,{ }^{* * *} p<0.0001$, difference & between \\
active and placebo rates.
\end{tabular}

the EWPHE trial are also given in Table 1. Important consequences of diuretic treatment included gout 4/1,000 patient years and diabetes mellitus $9 / 1,000$ patient years (13). The latter was associated with starting oral hypoglycaemic drugs in $7 / 1,000$ patient years. Active treatment was also associated with a need for peripheral vasodilators in $7 / 1,000$ patient years (14). Table 1 also indicates that a certain degree of mild hypokalaemia and an elevated serum creatinine will be expected in actively treated patients. As deaths from cardiac causes and renal failure were not increased on active treatment these biochemical changes were presumably of no clinical importance. An excess of $12 \%$ of patients reported dry mouth and $7 \%$ diarrhoea. This appeared to be mainly due to the methyldopa treatment received by a third of patients on active treatment. Examining Table 1, most would agree that the prevention of cardiac and stroke deaths and prevention of non fatal stroke and severe congestive heart failure were benefits that far out-weigh the disadvantages or risks of treatment.

Quality of life (QOL) was not formerly measured in the EWPHE trial and was probably impaired to some extent in the actively treated group owing to the fact that a third received methyldopa. However, a non fatal stroke or severe heart failure would have dramatically reduced QOL in the placebo treated group.

One would expect that, in survivors with no complication of hypertension, placebo would be associated with the best QOL. However, a recent report from the Treatment of Mild Hypertension Study (TOMHS) stated that two treatments were associated with a statistical improvement in QOL over placebo, namely acebutolol $(p=0.007)$ and chlorthalidone $(p=0.007)$ and that amlodipine, doxazosin and enalapril occupied intermediate positions (15). This surprising finding, that QOL was least good on placebo may have been due to the subjects being aware of their blood pressure and the fall in blood pressure being least in the placebo group. Nevertheless, the TOMHS is useful in comparing five active treatments and a simple analysis of the QOL data is presented in Table 2. This analysis suggests that QOL may be less acceptable on doxazosin and the authors report that this drug was worse than acebutolol and chlorthalidone for both energy and general functioning $(p<0.01$ for both comparisons), and also worse than amlodipine for general functioning alone. Enalapril was also no better than placebo. A recent trial comparing prazosin with six other treatments (16) found a withdrawal rate due to adverse drug reactions of $14 \%$ on prazosin, $10 \%$ on clonidine, $6-7 \%$ on placebo and diltiazem, $5 \%$ on captopril and $2 \%$ and $1 \%$ on atenolol and hydrochlorothiazide. Withdrawal rates are related to QOL (17) and it must be concluded that a impairment of QOL may occur with the alpha adrenoceptor blocking drugs. Five drugs have been associated with a good QOL, atenolol, captopril, enalapril, cilazapril and verapamil and two drugs are associated with a less good QOL, propranolol and nifedipine (17). In Table 3, three drugs are 
Table 2. Percentage Change ( + , Improvement) in 7 Aspects of Quality of Life Examined in the Treatment of Mild Hypertension Study (TOMHS)

\begin{tabular}{|c|c|c|c|c|c|c|}
\hline & Acebutolol & Amlodipine & Chlorthalidone & Doxazosin & Enalapril & Placebo \\
\hline General health index & +6.7 & +2.0 & +4.6 & +4.8 & +3.9 & +4.1 \\
\hline Energy/fatigue & +9.4 & +4.5 & +10.0 & +3.4 & +5.8 & +5.2 \\
\hline Mental health index & +4.7 & +3.2 & +4.3 & +2.2 & +2.1 & +2.3 \\
\hline General functioning index & +2.9 & +2.1 & +2.9 & -2.1 & -0.7 & -1.4 \\
\hline Satisfaction with physical abilities & +13.6 & +14.0 & +13.3 & +6.7 & +8.9 & +8.9 \\
\hline Social functioning & +2.8 & +5.4 & +2.8 & 0 & +5.4 & 0 \\
\hline Social contacts & 0 & +5.4 & -3.4 & 0 & -1.7 & +3.5 \\
\hline Average & +5.7 & +5.2 & +4.9 & +2.1 & +3.4 & +3.2 \\
\hline
\end{tabular}

Table 3. Relationship between Some Anti-Hypertensive Drugs and Measures of Quality of Life (QOL)

\begin{tabular}{ll}
\hline Drugs associated with a good QOL \\
Atenolol, acebutolol (beta blockers) \\
ACE inhibitors: captopril, enalapril, cilazapril \\
Verapamil, amlodipine (calcium channel blockers) \\
Diuretics \\
Drugs that have been proven to impair certain aspects \\
of QOL \\
Methyldopa \\
Propranolol & (centrally acting drug) \\
Nifedipine & (beta blocker) \\
? Doxazosin & (calcium channel blocker) \\
\hline
\end{tabular}

added, as a result of the TOMHS, acebutolol, amlodipine and diuretics for their association with a good QOL. Methyldopa is also added as a drug associated with poor QOL (18). In this table it is assumed that all ACE inhibitors are the same. However, a recent trial compared captopril and enalapril and concluded that "patients treated with captopril had more favourable changes in overall quality of life, general perceived health, vitality, health status and emotional control" (19). This was surprising as side effects were similar in the two groups (Table 4) and moreover, the withdrawal rates were almost identical on the two drugs. In the event, the statistically significant result occurred mainly with the General Perceived Health Scale (Table 5) although there was some support from the emotional control scale and the Side Effects and Symptoms Distress Index (Table 6). TOMHS listed 53 symptoms and when considering the five active treatments, enalapril should have had the highest complaint rate, by chance, in 11 . In the event enalapril was associated with the highest complaint rate in 19 symptoms, most notably lightheadedness when standing up. However, a preference for captopril over enalapril was not demonstrated in an earlier study (20) where general health (from the Psychological General Well-being scale) improved more on enalapril than captopril $(p<0.05)$.

\section{Costs}

The costs of primary stroke prevention are mea-
Table 4. Side Effects and Withdrawal Rates on Two ACE Inhibitors (Testa et al, 19)

\begin{tabular}{lcc}
\hline & Captopril & Enalapril \\
\hline Headache (\%) & 15 & 16 \\
Asthenia (\%) & 9 & 10 \\
Dizziness (\%) & 8 & 7 \\
Cough (\%) & 5 & 6 \\
\hline Withdrawal due to & & \\
$\quad$ adverse events (\%) & 9 & 8 \\
Total withdrawals (\%) & 21 & 20 \\
\hline
\end{tabular}

sured by cost-effectiveness, where the costs are divided by a health effect; or by cost utility where costs are divided by quality of life adjusted units of survival (QALYS). The most important health effect that has been studied is the number of years of life gained by the expenditure of money. There are no health effects that have been studied that are specifically concerned with benefits derived solely from stroke prevention. Most studies have utilised data from Western societies and thus the prevention of death from ischaemia heart disease has had a major effect on the calculations. In societies where stroke is more common than heart disease, the costeffectiveness analysis would be more relevant to stroke prevention per se.

The major difficulty in cost-effectiveness analysis has been in determinig the life years gained. Extrapolation from epidemiological studies has not provided the correct denominator. However, the estimates of cost-effectiveness are improving with the recent meta-analysis of the major trials providing estimates of the Fraction of Benefit (FOB) to be achieved. It does now appear possible to calculate the cost of an extra year of life, although these costs do vary according to health care system and the drugs used. The cost of an extra year of life are lower at higher levels of pressure, in men rather than women and at older ages (21). It is very difficult to examine other conditions to see if the treatment of, say, hypercholesterolaemia or non-insulin dependent diabetes mellitus costs less to achieve one extra year of life. Those who have considered the costs of treating mild hypertension have guessed whether we get "value for money" or not. The costeffectiveness of treating diastolic pressures over 
Table 5. Quality of Life Changes on Two ACE Inhibitors (Testa et al, 19)

\begin{tabular}{|c|c|c|c|}
\hline & Captopril & Enalapril & $p$ \\
\hline \multicolumn{4}{|l|}{ Psychological Distress Scale } \\
\hline Anxiety & - & +0.11 & NS \\
\hline Depression & - & - & NS \\
\hline Behaviourial or emotional control & +0.15 & +0.10 & 0.029 \\
\hline Total & - & - & NS \\
\hline \multicolumn{4}{|l|}{ Psychological Well-being Scale } \\
\hline General positive effect & +0.20 & - & NS \\
\hline Emotional ties & +0.18 & +0.14 & NS \\
\hline Life satisfaction & - & - & NS \\
\hline Total & +0.24 & +0.12 & NS \\
\hline \multicolumn{4}{|l|}{ General Perceived Health Scale } \\
\hline Vitality & +0.15 & -0.20 & 0.007 \\
\hline General health status & - & -0.20 & 0.011 \\
\hline Sleep disturbance & - & - & 0.015 \\
\hline Total & +0.12 & -0.21 & $<0.001$ \\
\hline
\end{tabular}

+ , improvement

Table 6. Quality of Life Changes on Two ACE Inhibitors (19)

\begin{tabular}{lccc}
\hline & Captopril & Enalapril & $p$ \\
\hline Well-being at work or in daily routine scale & 0 & -0.15 & 0.082 \\
Sexual symptom distress & -0.19 & -0.18 & $\mathrm{NS}$ \\
Side effects and symptoms distress index & +0.10 & -0.08 & 0.068 \\
Overall quality of life & +0.11 & -0.11 & $<0.05$ \\
\hline
\end{tabular}

$100 \mathrm{mmHg}$ appears not to be excessive (21). A measure of cost-effectiveness does not take into account the QOL during treatment and this adjustment has been essential with certain anti-hypertensive treatments employed in the past. It is obviously important to consider both quantity and quality of life and QOL can be measured by collecting standard data on many aspects of well-being (physical and psychological) and activity. However, such measures have to be aggregated to give a Health Status Index which is utilised to adjust the years of survival and give the required QALYS. This is sensible in a hierarchical sense but does not necessarily allow us to compare, say, hip replacement with antihypertensive treatment. Firstly the studies have not been performed to compare such disparate conditions and secondly the numeric results will depend heavily on what is included in any assessment and the weightings given to various aspects of life. The corrections for QOL with anti-hypertensive treatment can render treatment undesirable. Although this may be true in certain circumstances, it would appear intuitively unlikely for severe hypertension. Fletcher has stated "there are sufficient problems with the current methodology to suggest we should not be using QALYS to determine treatment options" (22). Acceptance of this view does not imply that QOL cannot be measured and compared within, for example, a double blind randomised controlled trial. If such a trial includes an overall measure of QOL, such as a HSI, mortality as an outcome, costs and different randomised treatments then the trial would generate QALYS which could be employed to decide on treatment options. Unfortunately no trial has measured both survival and QOL carefully enough to allow this comparison. Long term randomised trials with mortality as an outcome should include a measure of QOL and costs and provide cost-effectiveness and cost-utility comparisons. In the meantime we can only assume that the drugs are similar in their effects on survival and then calculate the cost of obtaining an extra QALY. However we already know that drugs are not similar and that, in the elderly, diuretics may be preferred to beta blockers (7). In the meantime some argue that diuretics have the most favourable costutility, others that this is an attribute of selective beta blockers, calcium channel blockers, alpha blockers or ACE inhibitors. Cost-utility analysis await good data, information that is unlikely to be available for at least five years.

\section{References}

1. Collins R, Peto R, MacMahon S, et al: Blood pressure, stroke, and coronary heart disease. Lancet 1990; 335: 827-838.

2. Amery A, Staessen J, Fagard R, van Hoof R: Hypertension in the elderly, in Buhler FR, Laragh $\mathrm{JH}$ (eds). Handbook of Hypertension Vol 13. The 
Management of Hypertension. Amsterdam, Elsevier 1990, pp 483-494.

3. Medical Research Council Working Party: MRC trial of treatment of mild hypertension: principle results. Br Med J 1985; 2: 97-104.

4. The IPPPSH Collaborative Group: Cardiovascular risk and risk factors in a randomised trial of treatment based on the beta blocker oxprenolol: the International Prospective Primary Prevention Study in Hypertension (IPPPSH). $J$ Hypertens 1985; 3: 379-392.

5. Wilhelmsen L, Berglund G, Elmfeldt D: Beta blockers versus diuretics in hypertensive men: main results from the HAPPHY trial. J Hypertens 1987; 5: 561-572.

6. Medical Research Council Working Party: MRC trial of treatment of hypertension in older adults: principle results. Br Med J 1992; 304: 405-412.

7. Beard K, Bulpitt C, Mascie-Taylor H, O'Malley K, Sever P, Webb S: Management of elderly patients with sustained hypertension. Br Med J 1992; 304: 412-416.

8. Coope J, Warrender TS: Randomised trial of treatment of hypertension in elderly patients in primary care. $\mathrm{Br}$ Med J 1986; 293: 1145-1151.

9. Dahlof B, Lindholm LH, Hanson L, Schersten B, Ekbom T, Wester P-O: Morbidity and mortality in the Swedish trial in old patients with hypertension (STOP-Hypertension). Lancet 1991; 338: 1281-1285.

10. Management Committee: Treatment of mild hypertension in the elderly. Med J Aust 1981; ii: 398-402.

11. Amery A, Birkenhäger WH, Brixko P, et al: Mortality and morbidity results from the European working party on high blood pressure in the elderly trial. Lancet 1985 ; i: $1349-1354$.

12. SHEP Co-operative Research Group: Prevention of stroke by antihypertensive drug treatment in older persons with isolated systolic hypertension. JAMA 1991; 265: 3255-3264.
13. Fletcher AE, Amery A, Birkenhäger W, et al: Risks and benefits in the trial of European Working Party on High Blood Pressure in the Elderly. J Hypertens 1991; 9: 225-230.

14. Fletcher AE (on behalf of EWPHE): Adverse treatment effects in the trial of the European Working Party on High Blood Pressure in the Elderly. Am J Med 1991; 90 (suppl 3A): 42-44.

15. The Treatment of Mild Hypertension Research Group: The treatment of mild hypertension study: a randomised, placebo-controlled trial of a nutritional hygienic regimen along with various drug monotherapies. Arch Intern Med 1991; 151: 1413-1423.

16. Materson BJ, Reda DJ, Cushman WC, et al: Single drug therapy for hypertension in men: a comparison of six antihypertensive agents with placebo. $N$ Engl J Med 1993; 328: 914-921.

17. Bulpitt CJ, Fletcher AE: Quality of life evaluation of antihypertensive drugs. Pharmacoeconomics 1992; 2: 95-102.

18. Croog SH, Levine S, Testa MA, et al: The effects of antihypertensive therapy on the quality of life. $N$ Engl J Med 1986; 314: 1657-1664.

19. Testa MA, Anderson RB, Nackley JF, Hollenberg NK and the Quality of Life Hypertension Study Group: Quality of life and antihypertensive therapy in men: a comparison of captopril with enalapril. $N$ Engl J Med 1993; 328: 907-913.

20. Steiner SS, Friedhoff AJ, Wilson BL, Wecker JR, Santo J-A: Antihypertensive therapy and quality of life: a comparison of atenolol, captopril, enalapril and propranolol. J Human Hypertens 1990; 4: 217-225.

21. Kawachi I, Malcolm LA: The cost-effectiveness of treating mild to moderate hypertension: a reappraisal. J Hypertens 1991; 9: 199-208.

22. Fletcher AE: Pressure to treat and pressure to cost: a review of cost-effectiveness analysis. $J$ Hypertens 1991; 9: 193-198. 